\title{
Experimental and Simulation Study on Inductance and Equivalent Resistance using Equivalent Circuit Model and Finite Element Method
}

\author{
Teruyoshi Sasayama*a) $^{* a}$ Member, Yusuke Yanamoto** Member \\ Shunsuke Funaji** Non-member, Takahiro Ao ${ }^{* * * *} \quad$ Non-member
}

(Manuscript received Oct. 14, 2015, revised Feb. 18, 2016)

\begin{abstract}
A zone-control induction heating $(\mathrm{ZCIH})$ system heats workpieces using several induction coils. To design and control the ZCIH system, parameters such as the self/mutual inductance and self/mutual-equivalent resistance between coils should be obtained in advance. For this purpose, we have proposed a practical method called "multiport transformer model" to reduce the computation time in calculating these parameters. In this study, we compare the parametric result obtained by simulations using the multiport transformer model, with the result obtained through the finite element method (FEM) analysis results and experimental data to validate the computational accuracy of the multiport transformer model. The comparison result shows that the characteristics of the inductance and equivalent resistance calculated by the multiport transformer model are similar to those obtained from the FEM analysis and experimental result. This indicates that our proposed model can contribute to the design optimization and control of a ZCIH system.
\end{abstract}

Keywords: equivalent circuit model, equivalent resistance, finite element method, induction heating

\section{Introduction}

To ensure rapid and uniform heating of a workpiece, the induction heating methods by using multiple coils have been proposed $^{(1)-(10)}$. When multiple coils are used for induction heating, interference between coils should be taken into consideration. To overcome this issue, the zone-control induction heating $(\mathrm{ZCIH})$ system was developed ${ }^{(5)-(10)}$. This system has several coils in which each coil is connected to an independent inverter unit. The frequencies and phases of the currents are considered as equal to avoid interference between coils.

Because the ZCIH system has several coils in each "zone", mutual inductance and mutual-equivalent resistance appear between the coils ${ }^{(4)-(8)}$. Therefore, each coil current heats the workpiece not only in its corresponding zone but also in the other zones. Parameters such as the mutual inductance and mutual-equivalent resistance should be obtained in advance to control the currents while suppressing the interference between coils. Previously, a trial-and-error approach

a) Correspondence to: Teruyoshi Sasayama. E-mail: sasayama @ kyushu-u.ac.jp

* Department of Electrical and Electronic Engineer, Kyushu University

744, Motooka, Nishi-ku, Fukuoka 819-0395, Japan

** Railroad Products Company, Nabtesco Corporation JA Kyosai Bldg., 2-7-9, Hirakawacho, Chiyoda-ku, Tokyo 102-0093, Japan

*** Machinery \& System Hq., Mitsui Engineering \& Shipbuilding Co. Ltd.

3-16-3, Tamahara, Tamano 706-0014, Japan

**** Engineering Department, MES Power-Electronics Industry Co. Ltd.

3-1-1, Tama, Tamano 706-8651, Japan was employed to obtain the appropriate parameters by calculating using the finite element method (FEM) ${ }^{(11)}$. However, because of the required computational overhead, realizing real-time control of the ZCIH system using FEM is impractical.

Recently, we have proposed an equivalent circuit model called the "multiport transformer model" to reduce the computational cost of the parameters. We performed a comparison with the FEM results to determine its accuracy ${ }^{(12)}$.

In this paper, we compare the parametric result obtained by simulations using the multiport transformer model, that by FEM analysis, and the experimental data. In the multiport transformer model and the FEM, frequency characteristics are also obtained in order to discuss the difference of the parametric result between these simulations. Furthermore, the computation time were compared between the multiport transformer model and the FEM analyses.

As shown later, the calculation time of FEM is greater than $1 \mathrm{~min}$, whereas that of the multiport transformer model is less than $1 \mathrm{~ms}$. Therefore, the multiport transformer model is suitable for the design optimization and control of a ZCIH system in real time.

\section{Methods}

2.1 Experiment A picture of the induction-heating system used in the experiment is shown in Fig. 1, and the electric circuit of the measurement system is shown in Fig. 2. Each coil had nine turns. The workpiece was made of graphite and had a relative permeability of one. The thickness of the workpiece was approximately $2 \mathrm{~mm}$. The resistivity of the workpiece was $1200 \mu \Omega \cdot \mathrm{cm}$. Coil 1 was connected to a square-wave inverter (Fuji electric Co. Ltd., HFR7.0C11K-2), and coil 2 was open. Note that the 


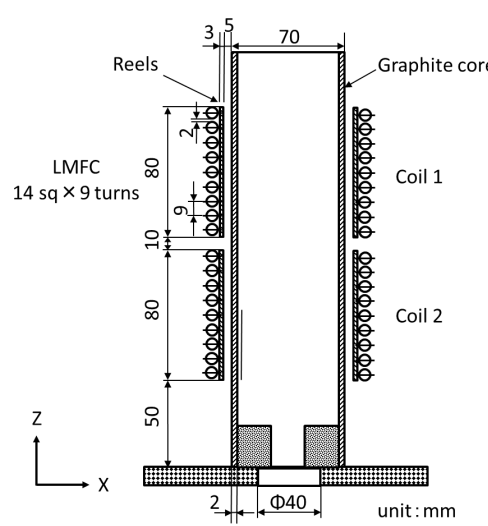

(a) Side view

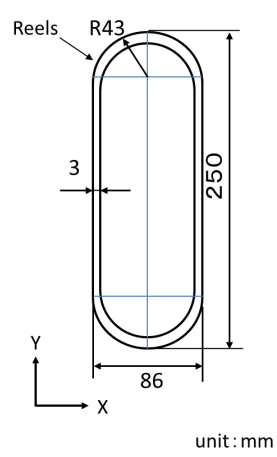

(b) Top view
Fig. 1. Dimension of the induction heating system

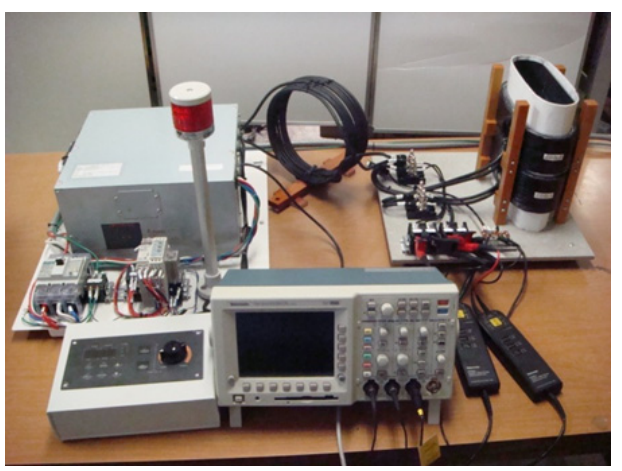

Fig. 2. Picture of the induction-heating system

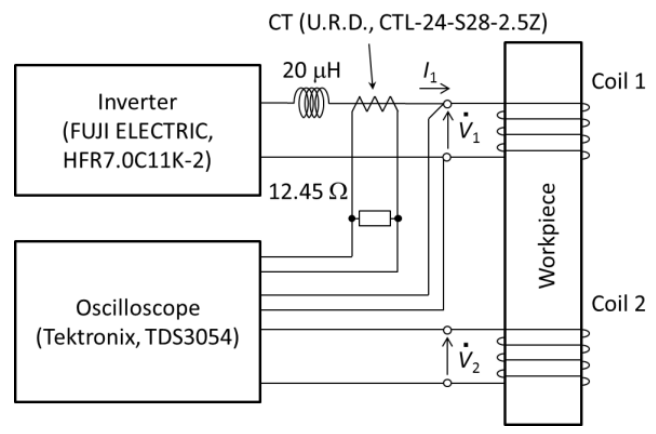

Fig. 3. Electric circuit of the measurement system

self/mutual inductance and self/mutual-equivalent resistance are not changed whether the current is fed into one of the coils or both two coils ${ }^{(10)(11)}$. Voltages $\dot{V}_{1}, \dot{V}_{2}$ and current $\dot{I}_{1}$ were measured using an oscilloscope (Tektronix Inc., TDS3054). The sampling rate was $100 \mathrm{MS} / \mathrm{s}$. The current and frequency of the inverter were set to $25 \mathrm{~A}_{\mathrm{rms}}$ and $39 \mathrm{kHz}$, respectively. The inverter output waveform was distorted because the output waveform contained not only the fundamental but also the harmonic waveforms. Therefore, a fast Fourier transform (FFT) was applied to the inverter output waveform for analysis. Table 1 lists the frequency component of the measured current.

By using induced voltage $\dot{V}_{n}$ and current $\dot{I}_{n}$ (angular frequency $\omega$ ) in coil $n$, the following equation is obtained:

$$
\dot{Z}_{n}=\frac{\dot{V}_{n}}{\dot{I}_{n}}=R_{n}^{*}+\mathrm{j} \omega L_{n}^{*}
$$

where $\mathrm{j}=\sqrt{-1}, R_{n}^{*}$ and $\omega L_{n}^{*}$ are the real and imaginary parts
Table 1. Frequency Component of the Current

\begin{tabular}{c|r|r}
\hline \hline Order & Frequency $[\mathrm{kHz}]$ & Current $\left[\mathrm{A}_{\mathrm{rms}}\right]$ \\
\hline 1st & 39.154 & 28.620 \\
3rd & 117.463 & 2.044 \\
5th & 195.771 & 0.688 \\
7th & 274.080 & 0.337 \\
\hline \hline
\end{tabular}

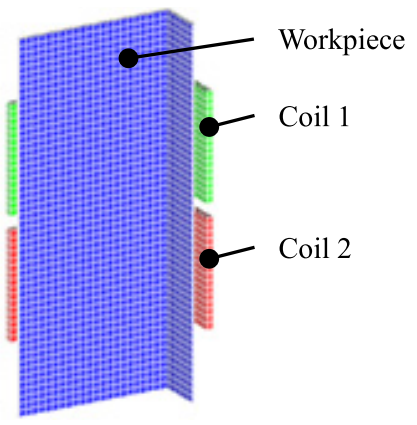

Fig. 4. A quarter of the region model

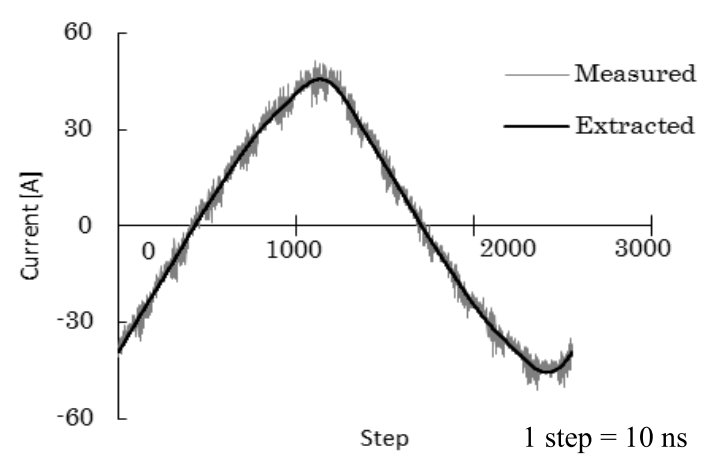

Fig. 5. Current waveform

of $\dot{Z}_{n}$, respectively, and the dot $(\cdot)$ above the variables represents a complex number. Using induced voltage $\dot{V}_{m}$ in coil $m$ and current $\dot{I}_{n}$ fed into coil $n$, we obtain

$$
\dot{Z}_{m, n}=\frac{\dot{V}_{m}}{\dot{I}_{n}}=R_{m, n}^{*}+\mathrm{j} \omega M_{m, n}^{*}
$$

where $R_{m, n}^{*}$ and $\omega M_{m, n}^{*}$ are the real and imaginary parts of $\dot{Z}_{m, n}$, respectively.

The procedure of calculating self/mutual inductance and self/mutual-equivalent resistance in this experiment are as follows. Substituting $\dot{V}_{1}$ and $\dot{I}_{1}$, which are measured by the oscilloscope, into Eq. (1), we can obtain $R_{1}^{*}$ and $L_{1}^{*}$. Substituting $\dot{V}_{2}$ and $\dot{I}_{1}$, which are also measured by the oscilloscope, into Eq. (2), we can obtain $R_{2,1}^{*}$ and $M_{2,1}^{*}$. Using the symmetry of the circuit, we can obtain $R_{2}^{*}=R_{1}^{*}, L_{2}^{*}=L_{1}^{*}, R_{1,2}^{*}=R_{2,1}^{*}$, $M_{1,2}^{*}=M_{2,1}^{*}$.

2.2 FEM Analysis Figure 4 shows an FEM model of the induction-heating system. The hexahedral elements were used in the FEM analysis, and the number of the elements is 165,168 .

The measured current waveform and the extracted waveform used for the FEM analysis are shown in Fig. 5. The fundamental, third-, fifth-, and seventh-harmonic components were extracted from the measured current waveform using FFT. The inductance and equivalent resistance were calculated with respect to each of the frequency components. 
In the FEM analysis, the time-stepping method (also called the step-by-step method) and the phasor method (also called the $\mathrm{j} \omega$ method) were applied using the extracted current, as shown in Fig. 5.

The governing equation of the eddy-current problem is

$$
\operatorname{rot}\left(\frac{1}{\mu} \operatorname{rot} \boldsymbol{A}\right)=\boldsymbol{J}_{0}-\sigma\left(\frac{\partial \boldsymbol{A}}{\partial t}+\operatorname{grad} \phi\right) .
$$

where $\mu$ is the permeability, $\boldsymbol{A}$ is the magnetic vector potential, $\boldsymbol{J}_{0}$ is the impressed current density, $\sigma$ is the conductivity, and $\phi$ is the electric scalar potential. In accordance with the experiment, $\mu$ and $\sigma$ were set to $4 \pi \times 10^{-7} \mathrm{H} / \mathrm{m}$ and $1200 \mu \Omega \cdot \mathrm{cm}$, respectively.

In the step-by-step method, the time of the one step was $1.0 \times 10^{-8} \mathrm{~s}$, and the number of the steps was 10,000 . Therefore, the period of the analysis was $1.0 \times 10^{-8} \times 10,000=$ $1.0 \times 10^{-4} \mathrm{~s}$. This is equivalent to approximately four-cycle times $\left(4 /\left(39 \times 10^{3}\right) \cong 1.0 \times 10^{-4} \mathrm{~s}\right)$. To reduce the computing time, the finite element meshes were partitioned into eight parts by using METIS ${ }^{(13)}$, and Eq. (3) was computed in parallel with Message Passing Interface (MPI).

The flux linkage $\Phi_{n}$ of coil $n$ is given by

$$
\Phi_{n}=\frac{N_{\mathrm{c}}}{S} \iiint_{\Omega} \boldsymbol{A} \cdot \boldsymbol{n}_{\boldsymbol{s}} \mathrm{d} \Omega, \cdot
$$

where $S$ is the cross-sectional area of the coil, $N_{\mathrm{c}}$ is the number of turns in the coil ( $\left.N_{\mathrm{c}}=9\right), \Omega$ is the volume of the coil, and $\boldsymbol{n}_{\mathrm{s}}$ is the unit vector parallel to the current.

The induced voltage $\dot{V}_{n}$ of coil $n$ is given by

$$
\dot{V}_{n}=-\mathrm{j} \omega \dot{\Phi}_{n}
$$

Finally, we can obtain $R_{m}^{*}, L_{m}^{*}, R_{m, n}^{*}$, and $M_{m, n}^{*}$ using (1), (2), and (5).

2.3 Multiport Transformer Model From the multiport transformer model shown in Fig. 6, we obtain the following voltage equations:

$$
\begin{aligned}
& \dot{\boldsymbol{V}}=\mathrm{j} \omega \boldsymbol{M} \dot{\boldsymbol{I}}+\mathrm{j} \omega \overline{\boldsymbol{M}} \dot{\overline{\boldsymbol{I}}} \\
& \dot{\overline{\boldsymbol{V}}}=\mathrm{j} \omega \overline{\overline{\boldsymbol{M}}} \dot{\overline{\boldsymbol{I}}}+\mathrm{j} \omega \overline{\boldsymbol{M}}^{\mathrm{T}} \dot{\boldsymbol{I}} \\
& \dot{\overline{\boldsymbol{V}}}=-\overline{\boldsymbol{R}} \dot{\overline{\boldsymbol{I}}} \ldots \ldots \ldots
\end{aligned}
$$

where

$$
\dot{\boldsymbol{V}}=\left[\begin{array}{ll}
\dot{V}_{1} & \dot{V}_{2}
\end{array}\right]^{\mathrm{T}}
$$

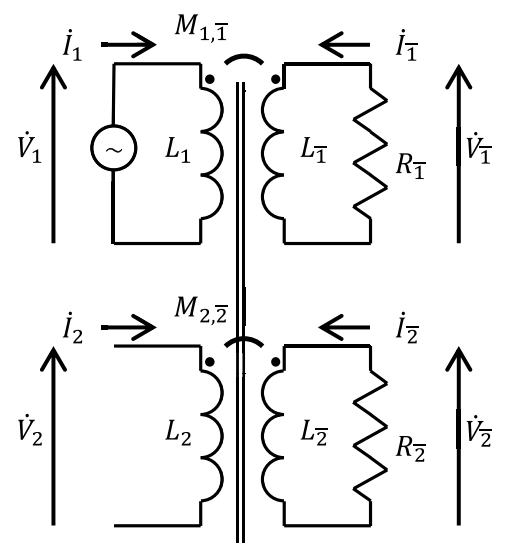

Fig. 6. Multiport transformer model (Two port)

$$
\begin{aligned}
& \dot{\overline{\boldsymbol{V}}}=\left[\begin{array}{ll}
\dot{V}_{\overline{1}} & \dot{V}_{\overline{2}}
\end{array}\right]^{\mathrm{T}} . \\
& \dot{\boldsymbol{I}}=\left[\begin{array}{ll}
\dot{I}_{1} & \dot{I}_{2}
\end{array}\right]^{\mathrm{T}} \cdots \\
& \dot{\bar{I}}=\left[\begin{array}{ll}
\dot{I}_{\overline{1}} & \dot{I}_{2}
\end{array}\right]^{\mathrm{T}} \ldots \\
& \boldsymbol{M}=\left[\begin{array}{cc}
L_{1} & M_{1,2} \\
M_{2,1} & L_{2}
\end{array}\right] \\
& \overline{\boldsymbol{M}}=\left[\begin{array}{ll}
M_{1, \overline{1}} & M_{1, \overline{2}} \\
M_{2, \overline{1}} & M_{2, \overline{2}}
\end{array}\right] \\
& \overline{\overline{\boldsymbol{M}}}=\left[\begin{array}{cc}
L_{\overline{1}} & M_{\overline{1}, \overline{2}} \\
M_{\overline{2}, \overline{1}} & L_{\overline{2}}
\end{array}\right] \\
& \overline{\boldsymbol{R}}=\left[\begin{array}{cc}
R_{\overline{1}} & 0 \\
0 & R_{\overline{2}}
\end{array}\right] \ldots
\end{aligned}
$$

where $\dot{V}_{m}$ and $\dot{V}_{\bar{m}}$ are the voltages of coil $m$ and workpiece $m$ (the workpiece under coil $m$ ), respectively. $\dot{I}_{m}$ and $\dot{I}_{\bar{m}}$ are the current of the coil and the workpiece, respectively, and $L_{m}$ and $L_{\bar{m}}$ are the DC inductance of coil $m$ and workpiece $m$, respectively. $M_{m, n}$ is the DC mutual inductance between coils $m$ and $n, M_{\bar{m}, \bar{n}}$ is the DC mutual inductance between workpieces $m$ and $n, M_{m, \bar{n}}$ is the DC mutual inductance between coil $\mathrm{m}$ and workpiece $n$, and $R_{\bar{m}}$ is the resistance of workpiece $m$. In the multiport transformer model analysis, we adopted the calculation algorithm for the DC self- and mutual inductance of thin-walled air coils ${ }^{(14)(15)}$. We note that the calculation algorithm can be applied only when the relative permeability of the workpiece is one.

By solving (6)-(8), we obtain the following equation:

$$
\dot{\boldsymbol{V}}=\left\{\mathrm{j} \omega \boldsymbol{M}+\omega^{2} \overline{\boldsymbol{M}}(\overline{\boldsymbol{R}}+\mathrm{j} \omega \overline{\overline{\boldsymbol{M}}})^{-1} \overline{\boldsymbol{M}}^{\mathrm{T}}\right\} \dot{\boldsymbol{I}}=\dot{\boldsymbol{Z}} \dot{\boldsymbol{I}} \cdots
$$

where

$$
\dot{\boldsymbol{Z}}=\mathrm{j} \omega \boldsymbol{M}+\omega^{2} \overline{\boldsymbol{M}}(\overline{\boldsymbol{R}}+\mathrm{j} \omega \overline{\overline{\boldsymbol{M}}})^{-1} \overline{\boldsymbol{M}}^{\mathrm{T}}
$$

Finally, we obtain $R_{m}^{*}, L_{m}^{*}, R_{m n}^{*}$, and $M_{m n}^{*}$ using $\dot{Z}$ as follows:

$$
\begin{aligned}
& R_{m}^{*}=\operatorname{Re}\left[\dot{Z}_{m, m}\right] \cdots \cdots \\
& L_{m}^{*}=\frac{1}{\omega} \cdot \operatorname{Im}\left[\dot{Z}_{m, m}\right] \cdots \\
& R_{m, n}^{*}=\operatorname{Re}\left[\dot{Z}_{m, n}\right], \\
& M_{m, n}^{*}=\frac{1}{\omega} \cdot \operatorname{Im}\left[\dot{Z}_{m, n}\right],
\end{aligned}
$$

where $\dot{Z}_{m n}$ is the matrix element of $\dot{Z}$.

The frequency characteristics of $R_{m}^{*}, L_{m}^{*}, R_{m, n}^{*}$, and $M_{m, n}^{*}$ were also analyzed in the simulation.

\section{Results}

Figure 7 shows the induced voltages of measurement and FEM simulation of the step-by-step method. A fairly good agreement between the measurement and FEM simulation is obtained (Correlation coefficient $>0.95$ ).

Table 2 shows the frequency components of the voltages shown in Fig. 7. Although the experimental result of the fundamental voltage is higher by approximately $10 \%$, a fairly good agreement between the measurement and FEM simulation is obtained.

Figure 8 shows the frequency characteristic result of $R_{m}^{*}$, 


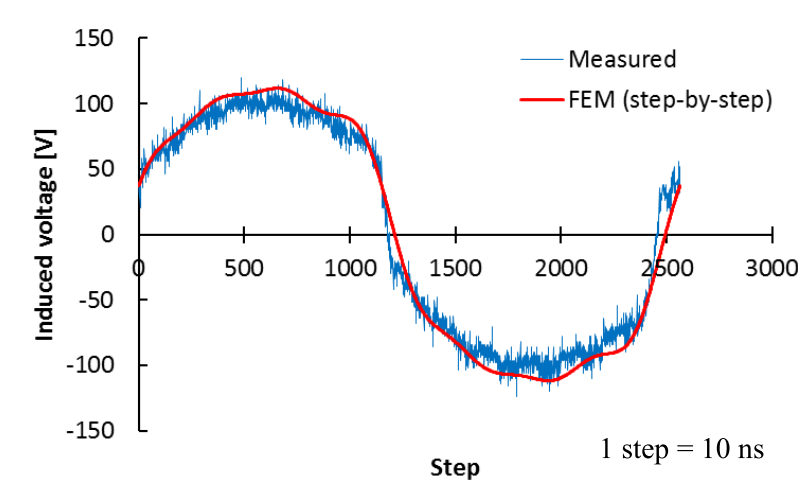

(a) Coil 1

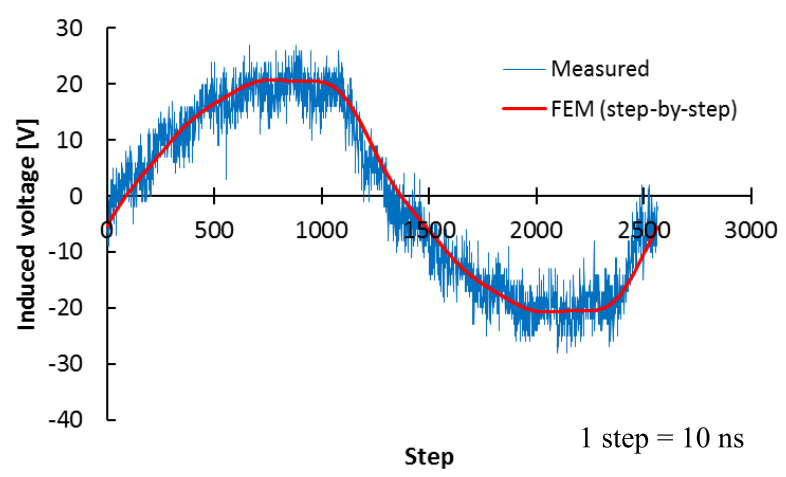

(b) Coil 2

Fig. 7. Induced voltage

Table 2. Frequency Component of the Voltage

\begin{tabular}{c|r|r|r|r}
\hline \hline \multirow{2}{*}{ Order } & \multicolumn{2}{|c|}{ Experiment $\left[\mathrm{V}_{\mathrm{RMS}}\right]$} & \multicolumn{2}{c}{ FEM analysis $\left[\mathrm{V}_{\mathrm{RMS}}\right]$} \\
\cline { 2 - 5 } & \multicolumn{1}{|c|}{ Coil 1 } & \multicolumn{1}{c}{ Coil 2 } & \multicolumn{1}{c}{ Coil 1 } & Coil 2 \\
\hline \hline 1st & 79.6 & 14.9 & 88.1 & 15.7 \\
3rd & 12.4 & 1.6 & 11.8 & 1.2 \\
5th & 6.7 & 0.77 & 6.2 & 0.58 \\
7th & 4.5 & 0.46 & 4.9 & 0.44 \\
\hline \hline
\end{tabular}

$L_{m}^{*}, R_{m, n}^{*}$, and $M_{m, n}^{*}$ calculated by FEM using the $\mathrm{j} \omega$ method and the multiport transformer model. A fairly good agreement between the FEM and multiport transformer model is obtained, especially when the frequency is less than or equal to $39 \mathrm{kHz}$ (the errors of estimated self/mutual-equivalent resistances by using the multiport transformer model are within $10 \%)$.

Figure 9 shows the results obtained from both experiment and computer simulation. For the fundamental harmonic, the characteristics of the inductance and equivalent resistance calculated by the multiport transformer model are similar to those obtained from the experimental result (the errors of estimated parameters by using the multiport transformer model are within approximately 20\%). This trend is also true for the harmonics except for the equivalent resistance of the seventhorder harmonic current.

Here, we discuss about the difference between the inductances estimated by the FEM and the multiport transformer model. Even though the frequency is relatively low $(\leq 39 \mathrm{kHz})$, the estimation error appears by approximately $20 \%$. This difference is considered to be caused by the approximation of the model in the multiport transformer model. In this model, DC self- and mutual inductances is calculated
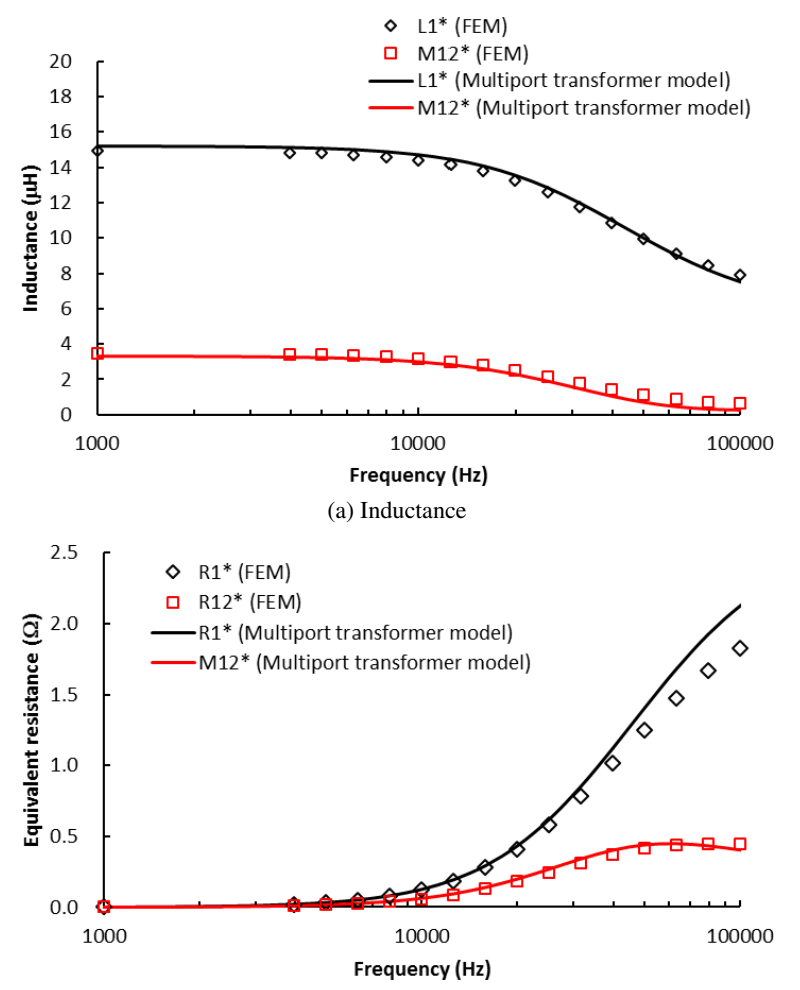

(b) Equivalent resistance

Fig. 8. Frequency characteristics of the circuit parameter

on the assumption that the coil and workpieces are sufficiently thin ${ }^{(14)(15)}$. If we use accurate values of the DC selfand mutual inductances in the multiport transformer model, the estimation error will decrease. Note that there is the tradeoff between the accuracy of the calculation and the calculation time.

Next, we discuss about the difference between the experimental and simulation results in the seventh-order harmonic current. One of the reasons of the difference is consider to be caused by skin effect. First, skin effect appears in the workpiece. Assuming that a relative permeability and the resistivity of the workpiece was one and $1200 \mu \Omega \cdot \mathrm{cm}$, respectively, the skin depths of the workpiece at the fundamental, 3th, 5th, and 7th harmonic components are $8.8 \mathrm{~mm}, 5.1 \mathrm{~mm}, 3.9 \mathrm{~mm}$, and $3.3 \mathrm{~mm}$ respectively. Because the thickness of the workpiece is approximately $2 \mathrm{~mm}$, the skin effect of the 7 th and higher harmonics strongly appears. In contrast, the skin effect is ignored in the FEM and the multiport transformer model. Second, skin effect also appears in the coil. The coil resistance itself increases due to the effect. In contrast, in this study, the coil resistance assumed to be zero. To calculate the equivalent resistances more accurately at higher frequency, the skin effect should be taken into consideration.

The other reason is due to the resolution of the measurement system. As shown in Table 1, the ratio of the $7 \mathrm{th} / 1 \mathrm{st}$ harmonic currents was relatively small $(0.337 / 28.620 \cong 0.01)$, whereas the resolution of the oscilloscope that we used in this study was 9 bits. Thus, the measurement error at the 7 th harmonic current would be large. To improve the accuracy of the measurement of the circuit parameters at higher frequency, the measurement resolution of the current and the voltage should be higher. 


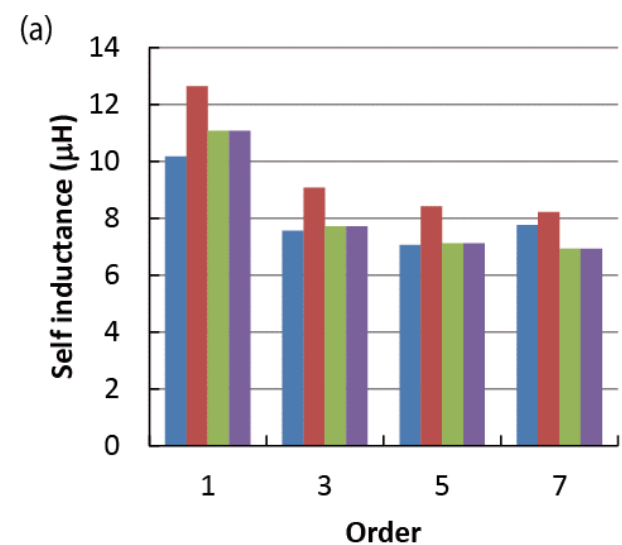

(c)

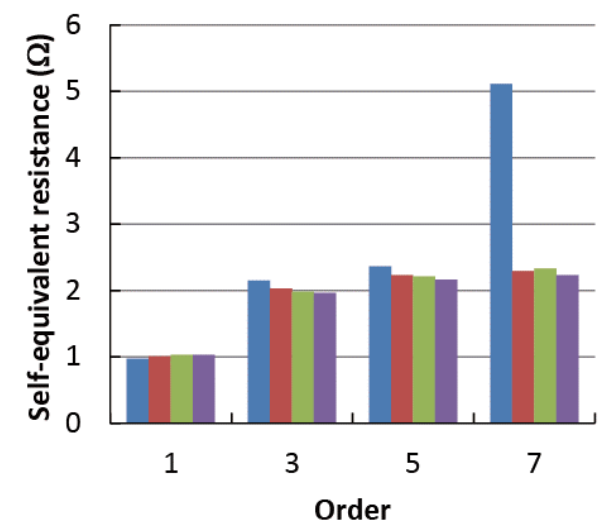

Fig. 9. Results of inductance and equivalent resistance: equivalent resistance, and (d) mutual-equivalent resistance

(b)
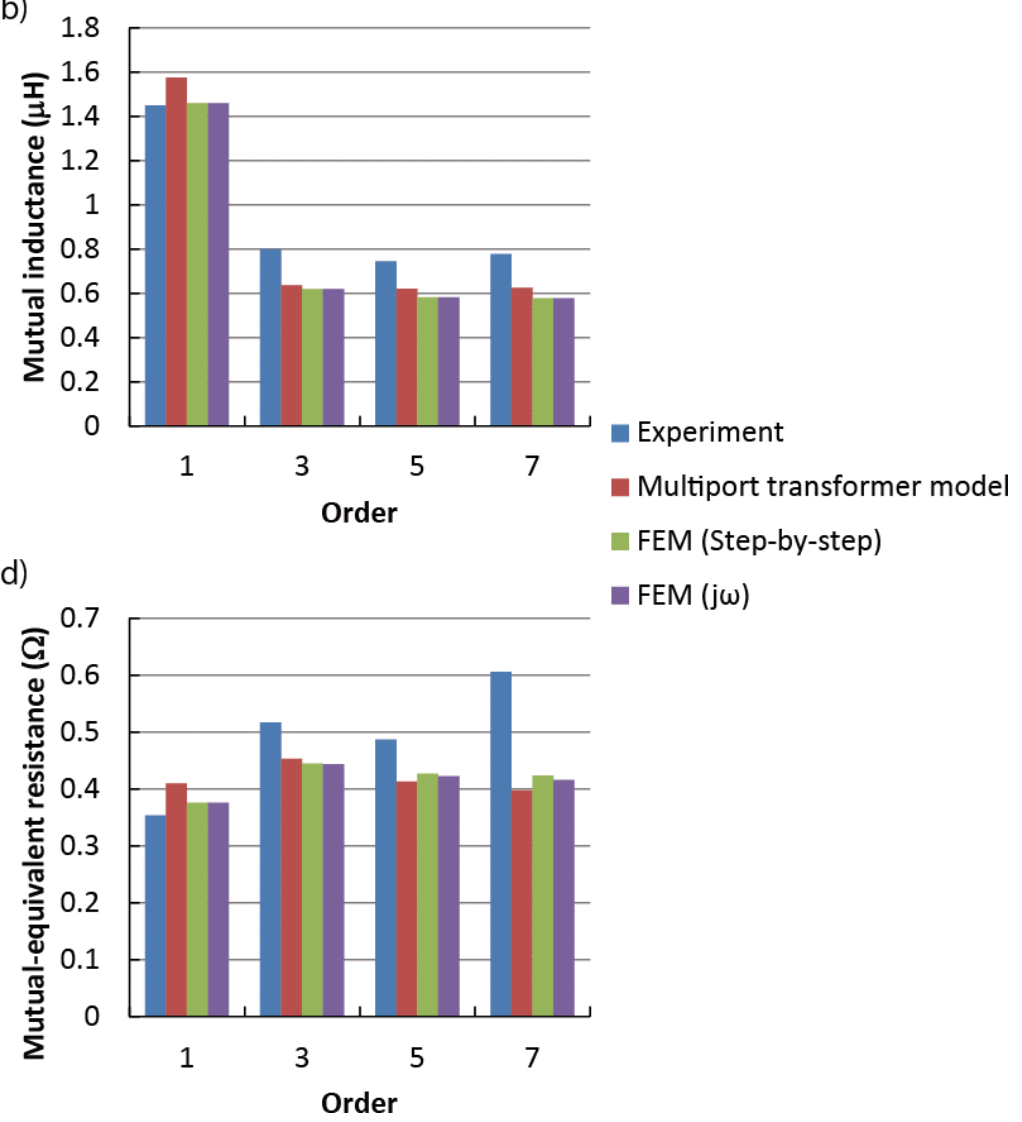

(a) Self-inductance, (b) mutual inductance, (c) self-

\section{Acknowledgment}

As shown in Fig. 9, the values calculated using the step-bystep and the $\mathrm{j} \omega$ methods are almost identical to those experimentally obtained because the permeability of the workpiece is unity. Therefore, in this situation, the $\mathrm{j} \omega$ method is useful to reduce the calculation time in the FEM analysis.

Using a computer (CPU: Intel Xeon Processor X5570 $2.93 \mathrm{GHz}$ with two processors; Memory: $12 \mathrm{~GB}$ ), the calculation time using FEM is approximately $12 \mathrm{~min}$ by the $\mathrm{j} \omega$ method (at each frequency component) and $800 \mathrm{~min}$ by the step-by-step method. In contrast, except for the calculation time of the DC self- and mutual inductances, the calculation time using the multiport transformer model is less than $1 \mathrm{~ms}$. Therefore, the multiport transformer model can greatly contribute to the design optimization and control of the $\mathrm{ZCIH}$ system in real time.

\section{Conclusion}

In this study, we have compared the circuit parameters $R_{m}^{*}$, $L_{m}^{*}, R_{m n}^{*}$, and $M_{m n}^{*}$ obtained by simulations using the multiport transformer model, FEM, and experimental data to validate the computational accuracy of the proposed multiport transformer model. The results show that $R_{m}^{*}, L_{m}^{*}, R_{m, n}^{*}$, and $M_{m, n}^{*}$ calculated by the multiport transformer model are almost identical to those calculated by FEM. Furthermore, for the fundamental harmonic, the characteristics of the inductance and equivalent resistance calculated by the multiport transformer model are similar to those obtained from the experimental result. Our proposed model will contribute to the design optimization and control of the ZCIH system.
This work was a collaborative effort between Mitsui Engineering \& Shipbuilding Co. Ltd. and Okayama University. The authors sincerely appreciate the excellent instruction and kind support offered by the late Prof. Norio Takahashi of Okayama University. We also wish to thank Mr. Naoki Uchida, a retired researcher of Mitsui Engineering \& Shipbuilding Co. Ltd., for his useful advice.

\section{References}

( 1 ) O. Lucía, J.M. Burdío, L.A. Barragán, J. Acero, and I. Millán: "Seriesresonant multiinverter for multiple induction heaters", IEEE Trans. Power Electron., Vol.15, No.2, pp.223-230 (2000)

( 2 ) F. Forest, E. Labouré, F. Costa, and J.Y. Gaspard: "Principle of a multiload/single converter system for low power induction heating", IEEE Trans. Power Electron., Vol.15, No.2, pp.223-230 (2000)

( 3 ) J.I. Rodriguez and S.B. Leeb: "Nonresonant and resonant frequencyselectable induction-heating targets", IEEE Trans. Power Electron., Vol.57, No.9, pp.3095-3108 (2010)

( 4 ) M. Souley, S. Caux, O. Pateau, P. Maussion, and Y. Lefévre: "Optimization of the settings of multiphase induction heating system", in Proc. IEEE Industry Application Society Annual Meeting, pp.1-6 (2012)

( 5 ) D. Miyagi, A. Saitou, N. Takahashi, N. Uchida, and K. Ozaki: "Improvement of zone control induction heating equipment for high-speed processing of semiconductor devices", IEEE Trans. Magn., Vol.42, No.2, pp.292-294 (2006)

( 6 ) H. Kurose, D. Miyagi, N. Takahashi, N. Uchida, and K. Kawanaka: "3-D eddy current analysis of induction heating apparatus considering heat emission, heat conduction, and temperature dependence of magnetic characteristics”, IEEE Trans. Magn., Vol.45, No.3, pp.1847-1850 (2009)

( 7 ) H. Kagimoto, D. Miyagi, N. Takahashi, N. Uchida, and K. Kawanaka: "Effect of temperature dependence of magnetic properties on heating characteristics 
of induction heater", IEEE Trans. Magn., Vol.46, No.8, pp.3018-3021 (2010)

( 8 ) H.N. Pham, H. Fujita, K. Ozaki, and N. Uchida: "Estimating method of heat distribution using 3-D resistance matrix for zone-control induction heating systems", IEEE Trans. Power Electron., Vol.27, No.7, pp.3374-3382 (2012)

( 9 ) H.N. Pham, H. Fujita, K. Ozaki, and N. Uchida: "Dynamic analysis and control for resonant currents in a zone-control induction heating system", IEEE Trans. Power Electron., Vol.28, No.3, pp.1297-1307 (2013)

(10) T. Sasayama, Y. Yanamoto, S. Funaji, and T. Ao: "Theoretical and numerical examination of equivalent resistances in zone-control induction heating system", IEEJ Ind. Appl., Vol.4, No.1, pp.67-73 (2015)

(11) Y. Yanamoto, T. Sasayama, N. Takahashi, N. Uchida, T. Ao, K. Kawanaka, and N. Matsunaka: "Examination on equivalent resistance and coupling coefficient of zone-control induction heating by finite element method", Proc. Compumag, PA6-16 (2013)

(12) T. Sasayama, Y. Yanamoto, S. Funaji, and T. Ao: "Practical method to calculate self- and mutual-inductance and equivalent resistance for a zone-control induction heating system", Proc. Intermag, AP-08 (2014)

(13) G. Karypis, et al., "METIS, Serial graph partitioning, version 5.0.2", http://www.cs.umn.edu//karypis/metis (2011)

(14) V. Pankrac: "The Algorithm for Calculation of the self and mutual inductance of thin-walled air coils of general shape with parallel axes", IEEE Trans. Magn., Vol.48, No.5, pp.1875-1889 (2012)

(15) V. Pankrac: "Generalization of relations for calculating the mutual inductance of coaxial coils in terms of their applicability to non-coaxial coils", IEEE Trans. Magn., Vol.47, No.11, pp.4552-4563 (2011)

Teruyoshi Sasayama (Member) was born in 1983. He received his

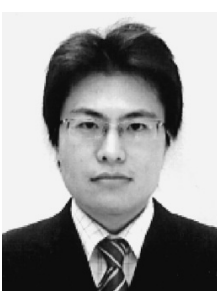
B.S. degree in electrical and electronic engineering in 2007, and his M.S. and Ph.D. degrees in electrical engineering in 2009 and 2012, respectively, from Kyoto University, Japan. He was an Assistant Professor in the Department of Electrical Engineering, Okayama University, Japan, from 2012 to 2014. Currently, he is an Assistant Professor in the Department of Electrical and Electronic Engineering, Kyushu University, Japan. His research interests include magnetic field analysis using the finite element method. Dr. Sasayama is a member of the Institute of Electrical and Electronics Engineers (IEEE), Institute of Electrical Engineers of Japan (IEEJ), and Institute of Electronics, Information and Communication Engineers (IEICE).
Yusuke Yanamoto (Member) was born in 1989. He received his

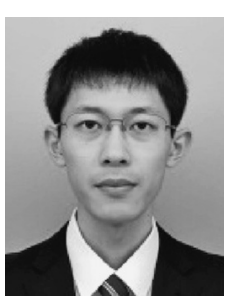
M.E. degree in Electronic and Information Systems Engineering from Okayama University in 2014. Mr. Yanamoto is a member of Institute of Electrical Engineers of Japan (IEEJ).

Shunsuke Funaji (Non-member) received the master's degree from

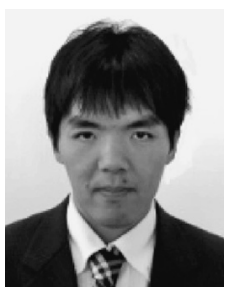
the Kanazawa University, Kanazawa, Japan in 2005. He joined the Mitsui Engineering \& Shipbuilding Co. Ltd., Tamano, Okayama, in 2005. He was involved in the development of various induction heating systems, mainly for automobile and steel industries, for 10 years.

Takahiro Ao (Non-member) received the master's degree from the

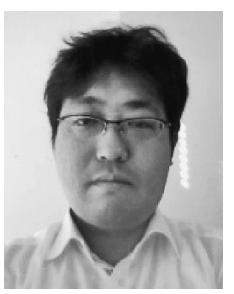
Tokushima University, Tokushima, Japan, in 1997. He joined the Mitsui Engineering \& Shipbuilding Co. Ltd., Tamano, Okayama, in 1997. He was involved in the development of various induction heating systems, mainly for automobile and steel industries, for 18 years. 